The Astrophysical Journal SuPPlement Series, 90:567-576, 1994 February

(C) 1994. The American Astronomical Society. All rights reserved. Printed in U.S.A.

\title{
TRANSPORT OF SOLAR ENERGETIC PARTICLES
}

\author{
WOLFGANG DRÖGE \\ Institut für Reine und Angewandte Kernphysik, Universität Kiel, Otto-Hahn-Platz 1, D-24118 Kiel, Germany \\ Received 1993 February 26; accepted 1993 June 9
}

\begin{abstract}
New developments in the understanding of the interplanetary transport of solar cosmic rays are reviewed. Based on carefully analyzed solar particle events observed on the Helios and ISEE 3 spacecraft, the relation of transport parameters to the structure of the interplanetary magnetic field is discussed. Special emphasis is given to a comparison of particle mean free paths determined from fits to intensity and anisotropy profiles with theoretical predictions derived from magnetic field spectra measured at the time of the solar particle event. Different aspects of the turbulence and wave models for the magnetic fluctuations are considered, including the effects resulting from the finite temperature of the plasma and of resonance broadening. It is found that a modified quasi-linear theory of particle scattering taking into account the effects of plasma waves propagating with respect to the average solar wind flow and the proper treatment of the dispersion relation at high wavenumber gives results which are in several cases in good agreement with particle observations in the interplanetary medium between 0.3 and $1 \mathrm{AU}$, indicating that quasi-linear theory is probably a good approximation to a full theory of solar particle transport. This has important implications for other astrophysical problems where quasi-linear theory is often used, such as the propagation and acceleration of Galactic cosmic rays and particle acceleration at shock waves.

Subject headings: acceleration of particles — cosmic rays - interplanetary medium - MHD — solar wind Sun: particle emission
\end{abstract}

\section{INTRODUCTION}

The scattering of cosmic rays in turbulent magnetic fields is a process which appears to operate in many astrophysical environments. Pitch-angle scattering by fluctuations imposed on the large-scale magnetic field, described by weak turbulence quasi-linear theory (QLT), is often assumed to be the basic physical process behind diffusive propagation of cosmic-ray particles in space plasmas. The results of QLT have been applied to many astrophysical problems, such as the propagation of cosmic rays in the interplanetary and interstellar medium and in supernova remnants, and the acceleration of energetic particles at shock waves. However, details of the fluctuation model assumed, the interpretation of the fluctuations either as waves or as dynamical turbulence, dissipation range and helicity effects, as well as others, are still under discussion. Especially, a long-standing problem was the observation that the theoretical particle mean free paths derived from the original QLT (Jokipii 1966; Hasselmann \& Wibberenz 1968) were considerably smaller than those derived phenomenologically from solar particle observations by fitting time-dependent diffusion models to measured particle time-intensity and time-anisotropy profiles (Palmer 1982; Kunow et al. 1991).

Over the years several improved approaches to QLT have been suggested. Whereas the original QLT derivation employed magnetostatic fluctuations in the slab model, in which wavevectors of the fluctuations are aligned with the mean magnetic field, models of oblique Alfvén waves (Lee \& Völk 1975; Jaekel \& Schlickeiser 1992) and of isotropic turbulence (Fisk et al. 1974; Smith, Bieber, \& Matthaeus 1990) have been studied. Moreover, in a separate development many attempts have been made to include nonresonant effects such as mirroring (Goldstein 1980; Davila \& Scott 1984) and resonance broaden- ing (Völk 1973) in the calculation despite the fundamental difficulty that they worsen the original discrepancy (Fisk 1979).

When it became evident from magnetometer and plasma wave observations in the solar wind (Coroniti et al. 1982; Denskat, Beinroth, \& Neubauer 1983) that the magnetic fluctuation spectra typically exhibit a dissipation range with a steepening of the spectrum above the ion gyrofrequency, the discrepancy problem between observed and theoretical mean free paths reversed: now the original QLT predicted mean free paths which were formally infinite due to a "resonance gap" at large pitch angles. As a consequence, higher order corrections to QLT become important to scatter particles through the resonance gap and yield mean free paths in agreement with observations. In addition to nonlinear and nonresonant processes mentioned above, wave propagation effects (Schlickeiser 1988) and the effects of dynamical turbulence (Bieber \& Matthaeus 1991) are among the proposed mechanisms.

Besides the extensive work on theoretical aspects of the transport of solar particles over the last 20 years, a large number of detailed observations from interplanetary missions such as the Helios and ISEE 3 (ICE) spacecraft have become available. As an important new finding, two recent studies (Wanner \& Wibberenz 1993; Wanner et al. 1993) have shown that the sizes of the discrepancy vary from event to event, and addressed attention to the need for an individual case-by-case analysis of each event for which the comparison of QLT and particle results is conducted.

The structure of this work is the following: in $\S 2$ a review of the phenomenological description of interplanetary transport of solar particles is given. Section 3 discusses the general background of the pitch-angle scattering problem. Section 4 gives a short overview of the nature of the interplanetary magnetic 
field fluctuations. In $\S 5$ a number of recently suggested improvements of quasi-linear theory are discussed. Section 6 summarizes the results and discusses possible implications for higher order corrections to the theory.

\section{TRANSPORT MODELS}

The interplanetary magnetic field can usually be described as a smooth average field, represented by an Archimedean spiral, with superposed irregularities. The motion of charged particles therefore consists of two components, adiabatic motion along the smooth field, and pitch-angle scattering off the irregularities. The quantitative treatment of the evolution of the particle's phase-space density $f(z, \mu, t)$ is given by the model of focused transport (Roelof 1969):

$$
\begin{aligned}
\frac{\partial f}{\partial t}+\mu v \frac{\partial f}{\partial z}+\frac{1-\mu^{2}}{2 L} v & \frac{\partial f}{\partial \mu} \\
& -\frac{\partial}{\partial \mu}\left[D_{\mu \mu}(\mu) \frac{\partial f}{\partial \mu}\right]=q(z, \mu, t),
\end{aligned}
$$

where $z$ is the distance along the magnetic field line, $\mu=\cos \theta$ is the particle pitch-angle cosine, and $t$ is the time. The particle velocity $v$ remains constant in this model, and motion perpendicular to the field is neglected. The systematic forces are characterized by $L(z)=B(z) /(-\partial B / \partial z)$, the focusing length in the diverging magnetic field $B$, while the stochastic forces are described by the pitch-angle diffusion coefficient $D_{\mu \mu}(\mu)$. The injection of particles close to the Sun is given by $q(z, \mu, t)$.

If the scattering is sufficiently strong, the distribution function $f(z, \mu, t)$ adjusts rapidly to quasi-equilibrium through pitch-angle diffusion, which is close to the isotropic distribution. In this case the particle scattering can be treated as spatial diffusion with an effective radial diffusion coefficient $K_{r}(r)=$ $\frac{1}{3} v \lambda_{r}$, with $\lambda_{r}$ the radial mean free path. This is equivalent to totally anisotropic diffusion along the spiral magnetic field with a parallel diffusion coefficient $K_{\|}=K_{r} / \cos ^{2} \varphi$, with $\varphi$ being the angle between the magnetic field and the radial direction (see Ng \& Gleeson 1975). Additional effects such as convection and adiabatic deceleration in the expanding solar wind can then be incorporated, and the transport equation can be written in the form (Parker 1965)

$$
\begin{aligned}
\frac{\partial U}{\partial t}+\frac{1}{r^{2}} \frac{\partial}{\partial r}\left(r^{2} V U-r^{2}\right. & \left.K_{r} \frac{\partial U}{\partial r}\right) \\
& -\frac{2 V}{3 r} \frac{\partial}{\partial E}(\alpha E U)=Q(r, E, t)
\end{aligned}
$$

Here $U(r, E, T)$ is the differential number density, $r$ is the radial distance from the Sun, $V_{\mathrm{SW}}$ is the solar wind velocity, and $\alpha=\left(E+2 E_{0}\right) /\left(E+E_{0}\right)$, where $E$ and $E_{0}$ are the kinetic and rest energy of the particles. The mean free path $\lambda_{\|}$which relates the pitch-angle scattering rate to the spatial diffusion parallel to the ambient magnetic field is given by (Hasselmann \& Wibberenz 1968; Earl 1973)

$$
\lambda_{\|}=\frac{3 v}{8} \int_{-1}^{+1} d \mu \frac{\left(1-\mu^{2}\right)^{2}}{D_{\mu \mu}(\mu)} .
$$

Because particle diffusion perpendicular to the average magnetic field in interplanetary space can be neglected (cf. Krimigis \& Roelof 1971), a perpendicular transport of particles close to the Sun is required to explain the azimuthal propagation of flare particles. This transport process has been named "coronal propagation." However, its details are still not well understood (for a review see Kunow et al. 1991). Most models assume that particles are accelerated on a short timescale in the impulsive, nonthermal phase of the flare, as indicated by hard X-ray and $\gamma$-ray observations. The particles then propagate in the coronal magnetic field structure which existed before the occurrence of the flare, or their lateral transport partly occurs by processes related to the flare, e.g., an expanding magnetic bottle. In a totally different approach (cf. Mason, Gloeckler, \& Hovestadt 1984), it is postulated that particles are not transported in the corona at all but are accelerated at a shock wave traveling through the corona and are released even far away from the flare site whenever the shock crosses interplanetary field lines leading out into interplanetary space. For the study of interplanetary transport the above processes are phenomenologically described by the injection function $Q(r, E, t)$ in equation (2).

Solutions of the transport equations ( 1 ) and (2) have been widely used to model observed intensity and anisotropy time profiles. Because analytical solutions in the general case are not known, numerical codes have to be used. An example, obtained with a code developed for the solution of equation (1) by Schlüter (1985) is shown in Figure 1. The mean free path obtained from the fit to the particle data, hereafter referred to as $\lambda_{\text {fit }}$, can then be compared with the value $\lambda_{\mathrm{th}}$ based on the

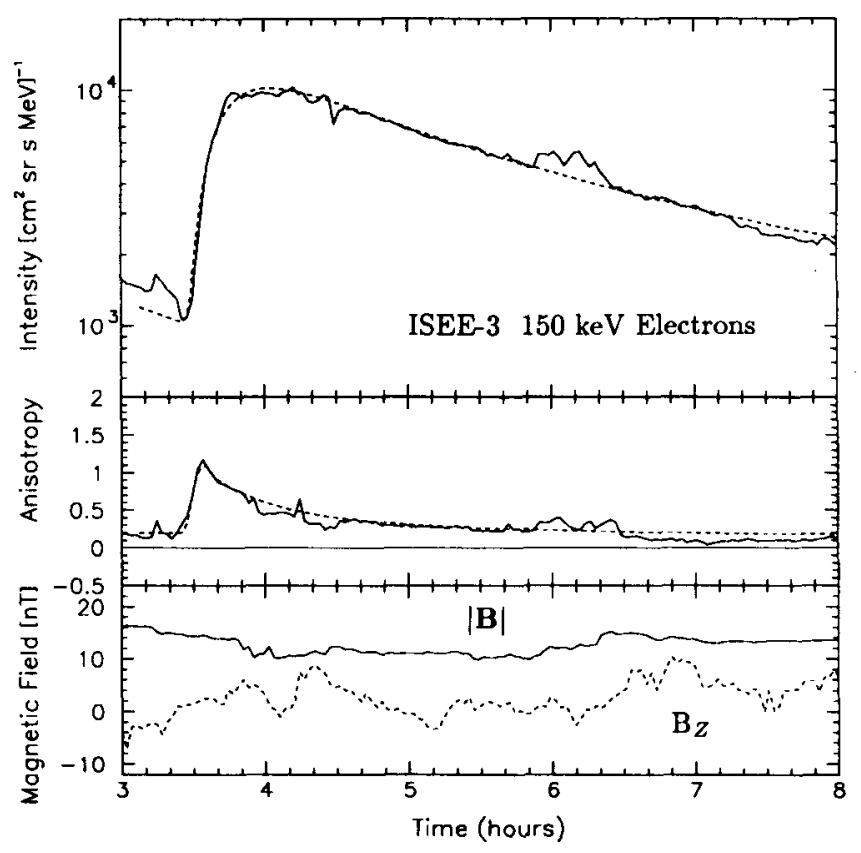

Fig. 1.-Particle and magnetic field observations of the 1980 June 7 event observed on ISEE 3. Electron data ( solid lines) can be fitted (dashed lines) in intensity ( upper panel) and anisotropy (middle panel) assuming a parallel mean free path of $\approx 0.4 \mathrm{AU}$. Simultaneous measurements of the magnetic field (lower panel) are utilized to monitor the propagation conditions and to determine fluctuation spectra of the magnetic field component perpendicular to average field $\left(B_{z}\right)$. 
theoretical derivation of $D_{\mu \mu}(\mu)$ from the observed power spectrum of the magnetic fluctuations. Successful fits to observed particle data often require a radial dependence of the mean free path, which is usually described by a power law, $\lambda_{r} \propto r^{b}$. The proper choice of $b$ is important in order to determine the correct local value of $\lambda$ at the point of observation. The radial dependence of $\lambda_{r}$ is not yet well determined and may vary considerably from one event to the next. Systematic multispacecraft studies where the spacecraft were located at different radial distances (Zwickl \& Webber 1977; Beeck et al. 1987) have led to values of $b$ between 0 and 0.7 . An extensive summary of near-Earth mean free paths $\lambda_{\|}$based on the analysis of carefully selected solar events was given by Palmer (1982). He found that values of $\lambda_{\|}$can vary by almost two orders of magnitude, but most of them are in a "consensus range" between 0.08 and 0.3 AU over a large range of rigidities, suggesting that there is no systematic dependence on rigidity. On the contrary, Beeck et al. (1987) report a power-law dependence of $\lambda$ with rigidity in the range from 30 to $600 \mathrm{MV}$ for selected events, where the exponents vary between 0.2 and 0.4 . As will be discussed later on, the variation of $\lambda$ with rigidity is particularly interesting because it can give important insights into the correct theoretical description of the scattering process. Additional information about the nature of the scattering coefficient can also be obtained from the analysis of pitch-angle distributions in the onset and maximum phase of the particle event. For a discussion of this method the interested reader is referred to the recent review by Kunow et al. (1991), which also includes many phenomenological results obtained from the Helios spacecraft which were particularly suited to the study of interplanetary propagation.

\section{THE PITCH-ANGLE DIFFUSION COEFFICIENT}

Cosmic-ray pitch-angle scattering is caused by irregularities of the magnetic field which violate the first adiabatic invariant. When the irregularities superposed on the average field are sufficiently small, a pitch-angle diffusion coefficient $D_{\mu \mu}(\mu)$ can be obtained in closed form by calculating first-order corrections to the particle's orbit in the average field, and ensemble averaging over the statistical properties of the turbulence (Jokipii 1966; for an introduction to the theoretical framework see, e.g., Fisk 1979). Small irregularities mean that the changes of the particle's pitch angle during a single gyration are small and many gyrations are required to change the pitch angle considerably, implying that the particles are scattered by irregularities which are in resonance with the particle gyration. As a cumulative result of many small random changes in its pitch angle, the particle experiences a macroscopic change in direction, leading to spatial diffusion along the field line. Because of the appearance of $D_{\mu \mu}(\mu)$ in the denominator of the integrand in the expression for $\lambda_{\|}$(eq. [3]), the behavior of the scattering rate at pitch angles where it is small has a disproportionate influence upon the mean free path. It can therefore be expected that $\lambda_{\|}$is regulated by the scattering rate at pitch angles where scattering is slowest, generally at pitch angles close to $90^{\circ}$.

If the turbulence is regarded as purely magnetic, the pitchangle diffusion coefficient can be written in the form (cf. Jae- kel \& Schlickeiser 1992)

$$
\begin{aligned}
D_{\mu \mu}(\mu)= & \frac{\Omega^{2}\left(1-\mu^{2}\right)}{2 B_{0}^{2}} \operatorname{Re} \sum_{n=-\infty}^{\infty} \int d^{3} k \int_{0}^{\infty} d \tau \\
& \times \exp \left[-i\left(k_{\|} v_{\|}+n \Omega\right) \tau\right] \\
& \times\left[J_{n+1}^{2}\left(\frac{k_{\perp} v_{\perp}}{\Omega}\right) P_{R R}(\boldsymbol{k}, \tau)+J_{n-1}^{2}\left(\frac{k_{\perp} v_{\perp}}{\Omega}\right) P_{L L}(\boldsymbol{k}, \tau)\right. \\
& -J_{n-1}^{2}\left(\frac{k_{\perp} v_{\perp}}{\Omega}\right) J_{n+1}^{2}\left(\frac{k_{\perp} v_{\perp}}{\Omega}\right) \\
& \left.\times\left[e^{2 i \psi} P_{R L}(\boldsymbol{k}, \tau)+e^{-2 i \psi} P_{L R}(\boldsymbol{k}, \tau)\right]\right],
\end{aligned}
$$

where $\Omega=e B_{0} / \gamma m c$ is the relativistic gyrofrequency of particles with mass $m$ and charge $e, B_{0}$ is the magnitude of the mean magnetic field, $\gamma$ is the usual Lorentz factor, and $\boldsymbol{k}$ is the wavevector. The correlation tensors are given by $\left\langle B_{\alpha}(\boldsymbol{k}, t) B_{\beta}^{*}\left(\boldsymbol{k}^{\prime}, t+\right.\right.$ $\tau)\rangle=\delta\left(\boldsymbol{k}-\boldsymbol{k}^{\prime}\right) P_{\alpha, \beta}(\boldsymbol{k}, t)$, where $\alpha, \beta$ denote Cartesian $(x, y, z)$ or helical $(L, R)$ coordinates and the assumption is made that fluctuations of different wavevectors are uncorrelated. Further reduction of the pitch-angle scattering coefficient can only be achieved if certain assumptions of the properties of the magnetic fluctuations are made, particularly the time dependence of the correlation functions to perform the $\tau$-integration. As it will turn out, the scattering rate of energetic particles is remarkably sensitive to the detailed properties of the fluctuations. A brief overview over the nature of the fluctuations in the solar wind will be given in the next section.

\section{INTERPLANETARY MAGNETIC FIELD FLUCTUATIONS}

A representative power spectrum of magnetic field fluctuations in the solar wind perpendicular to the average field is shown in Figure 2 (from Denskat et al. 1983), obtained from Helios 2 at a radial distance of $0.3 \mathrm{AU}$. In the inertial range extending from $10^{-4}$ to $0.1 \mathrm{~Hz}$ the spectra typically exhibit a moderately steep power law with the exponent varying from -1.5 to -1.9 , whereas the high frequencies in the so-called dissipation range above $1 \mathrm{~Hz}$ display a steepening of the spectrum with slopes of approximately -3 . One of the problems to apply quasi-linear theory to comparison with observations is that in order to determine the scattering coefficient the full knowledge of the correlation tensor of the fluctuation is required. However, magnetometer and plasma wave instruments on spacecraft measure at best a time series of the field vector along a line, or the power density of fluctuations in a certain direction. From such measurements alone the exact nature of the correlation tensor cannot be deduced, and assumptions on its three-dimensional structure have to be made.

Two approaches have dominated discussion of the nature of the fluctuations and, subsequently, their interaction with charged particles. One model, the turbulence model, describes the observed properties of the magnetic fluctuations under the assumptions that the fluctuations interact. In this picture there are no deterministic correlations between frequency and wavenumber, and the only adequate description is a statistical one. For strong turbulence the characteristic time of interaction is comparable to the wave period. In the second model, the wave 


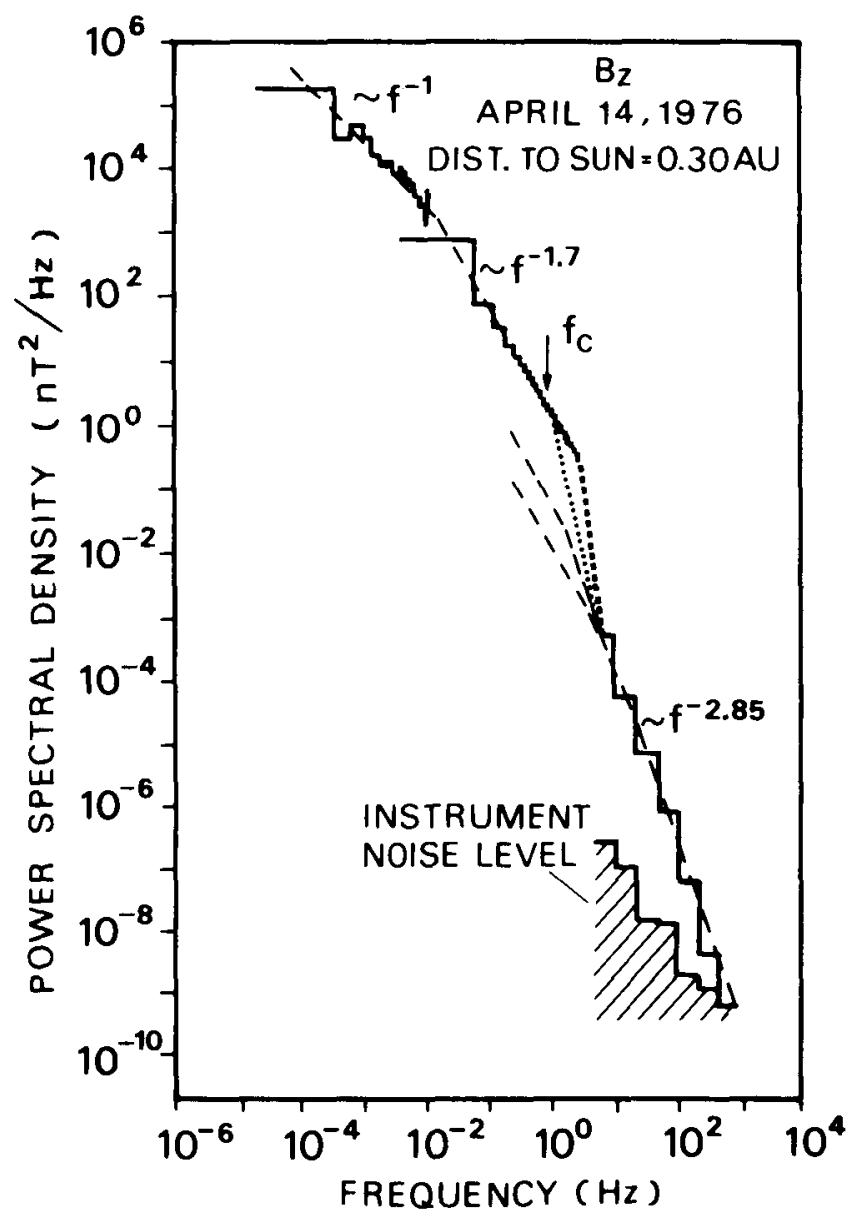

FIG. 2.-Power spectral density estimates of the magnetic field component $B_{z}$ observed on Helios 2 (from Denskat et al. 1983). The spectra typically exhibit a break around $1 \mathrm{~Hz}$, approximately the Doppler-shifted proton gyrofrequency.

model, a much more deterministic approach is considered. The magnetic fluctuations are described as superposition of small-amplitude plasma waves whose dispersion relations reflect the physical state of the wave-carrying background medium. Interaction between the waves is generally considered to be minimal. The solutions of the dispersion relation for parallel ( to $\boldsymbol{B}_{0}$ ) propagating waves in a cold plasma include several wave modes: at small wavenumbers $k \ll k_{c} \equiv 2 \Omega_{p} / V_{\mathrm{A}}$ and frequencies $\omega \ll \Omega_{p}$, the asymptotic dispersion relation is $\omega \simeq$ $V_{\mathrm{A}} k$, which describes nondispersive $\left(V_{\mathrm{Ph}}=\omega / k=\right.$ const.) Alfvén waves which are composed of either right-hand or lefthand circularly polarized waves. At large wavenumbers $k \gg k_{c}$, the left-hand Alfvén branch develops into the left-hand ion cyclotron wave branch, which at all values of $k$ has nearly the same frequency $\omega \simeq \Omega_{p}$. At large wavenumbers $k \gg k_{c}$ and frequencies in the range $\Omega_{p}<\omega<\left|\Omega_{e}\right|$, the right-hand Alfven branch develops into the right-hand whistler wave branch, which is dispersive ( $V_{\mathrm{Ph}} \neq$ const.), because of the quadratic wavenumber dependence of its dispersion relation $\omega \simeq 2 V_{\mathrm{A}} k^{2}$ / $k_{c}$. At very large wavenumbers $k \gg 21 k_{c}$, the right-hand whistler wave branch develops into the right-hand electron $c y$ - clotron wave branch $\omega \simeq \Omega_{e}$, which again can propagate both forward and backward. Wave modes propagating across the static magnetic field also exist.

From the observational side, correlations between the fluctuations in the solar wind velocity and the magnetic field component perpendicular to the average field in the inertial range are consistent with Alfvén waves traveling inward and outward with respect to the Sun (for a review on observations of turbulence in the solar wind see recent articles by Gurnett 1991 and Marsch 1991). However, the fact that the correlation is less than $100 \%$ indicates the additional presence of magnetoacoustic wave modes and/or static structures, and may also give evidence for nonlinear effects. Ion acoustic waves have been identified by independently measuring both the wavelength and the frequency. At high frequencies, correlations between the magnetic and electric field measurements indicate the existence of whistler waves (Coroniti et al. 1982). The break in the spectrum (cf. Fig. 2) is consistent with the expected damping of left-hand polarized Alfvén waves developing into the ion cyclotron branch as the frequencies approach the background plasma's proton gyrofrequency at $\simeq 1 \mathrm{~Hz}$, possibly somewhat stretched out in frequency due to Doppler shifts.

On the other hand, the amplitudes of the fluctuations can be quite large $\left(\delta B / B_{0} \approx 0.3\right)$, which indicates that significant nonlinear interaction could occur and make the concept of a superposition of small-amplitude waves rather questionable. An additional problem arises because in a pure wave picture the right-hand polarized Alfvén wave developing into the whistler branch is not affected by ion cyclotron damping. Observations show no evidence of persistent, nonzero magnetic helicity in the inertial range when statistically independent frequencies are averaged together (cf. Matthaeus \& Goldstein 1982). It is therefore reasonable to assume that waves of both polarization states are present with about equal intensities in the inertial range, and from Figure 2 it is evident that some dissipation mechanism must also act upon the right-hand waves. Presently it is not clear what the origin of this dissipation is. However, there are some hints from magnetohydrodynamic (MHD) theory for low-frequency fluctuations. Li \& Zweibel (1987), e.g., considered the scattering of Alfvén waves by density fluctuations and concluded that the interaction with density irregularities caused an energy transfer to magnetosonic waves and an attenuation of the original wave. Another possibility is the transfer of wave energy by nonlinear coupling between right-hand and left-hand waves (Dobrowolny, Mangeney, \& Veltri 1980). Probably the turbulence and wave pictures are not mutually exclusive but are inextricably linked. The resulting combination of both models must be able to explain the nonlinear evolution of the fluctuation spectra as a function of both the wavenumber and the radial distance from the Sun, and the various dissipation processes for the conversion of magnetic energy into particle energy, which include several resonances between the fluctuations and the ambient ion and electron population. It is quite possible that the evolution of the fluctuations is adequately described in the turbulence picture, whereas the interaction of the fluctuations with charged particles and therefore at least the qualitative features of $D_{\mu \mu}(\mu)$ and $\lambda_{\text {th }}$ can be understood from linear theory in the wave model. 


\section{COMPARISON BETWEEN THEORY AND OBSERVATIONS}

In the early work of Jokipii ( 1966) and Hasselmann \& Wibberenz (1968) the field irregularities were considered as magnetostatic, i.e., it was assumed that the observed fluctuations were "frozen" in the solar wind and convected past the spacecraft. As a consequence, the particles in the rest frame of the solar wind would sample a time-stationary magnetic field. In the case of wavevectors only parallel or antiparallel to the mean magnetic field (slab geometry), the pitch-angle diffusion coefficient (eq. [4]) reduces to the form

$$
D_{\mu \mu}(\mu)=\frac{\pi}{4} \frac{\Omega^{2}\left(1-\mu^{2}\right)}{B_{0}^{2} v|\mu|} P_{\perp}\left(k_{\text {res }}=\left|\frac{\Omega}{\mu v}\right|\right),
$$

where $P_{\perp}(k)$ is the (one-sided) total power spectrum of the fluctuations in both directions perpendicular to the mean magnetic field, evaluated at the resonant wavenumber. Fisk et al. (1974) found that the pitch-angle scattering coefficient in isotropic turbulence contains approximately one factor of $|\mu|$ more than the slab expression and can be approximated by $D_{\mu \mu}^{\text {iso }}(\mu) \approx|\mu| D_{\mu \mu}^{\text {si }}(\mu)$, when $\mu \neq 0$ and the power spectrum is not a steeply falling function of wavenumber.

Assuming slab geometry and a power spectrum $P_{1}(k) \propto k^{-q}$ extending to arbitrarily high wavenumbers leads to a $|\mu|^{q-1}$ variation of the scattering rate as the pitch angle approaches $90^{\circ}$. As long as $q<2$, this is slow enough that equation (3) leads to finite mean free paths which vary as $R^{2-q}$, where $R$ is the particle rigidity. For $q \geq 2$ the gap near $\mu=0$ becomes so large that particles are not scattered through pitch angle $90^{\circ}$ at all and no spatial diffusion is possible. Instead, the particles propagate in the so-called coherent propagation mode (Earl 1973), where the particle transport in the two pitch-angle hemispheres is decoupled. The isotropic model yields infinite mean free paths for $q \geq 1$ and hence for any spectra consistent with observations.

Observations show (Belcher \& Davis 1971; Daily 1973) that the power of the fluctuations in the field strength is considerably smaller than that of those in the field direction, and that a minimum-variance direction parallel to the large-scale magnetic field exists. This has generally been taken as evidence that the slab model is a good representation of the magnetic field fluctuations. Unfortunately, predictions from magnetostatic dissipationless QLT together with the slab model, which we will refer to as standard QLT hereinafter, are in poor agreement with particle observations. On average, the resulting values of $\lambda_{\text {th }}$ obtained from interplanetary magnetic field spectra are a factor of 10 too small compared with the ones deduced from observations of solar particle intensity profiles (cf. Palmer 1982). In a recent study Wanner et al. ( 1993) analyzed 29 solar events where fluctuation spectra and particles were observed simultaneously on board the Helios spacecraft, and compared mean free paths of $\approx 20 \mathrm{MeV}$ protons with the ones predicted by standard QLT. They find that the ratio between fit and standard QLT mean free paths (see Fig. 3) exhibits a large variation and can take on values between 0.5 and 25 . There seems to be no systematic variation of either $\lambda_{\text {th }}$ or $\lambda_{\text {fit }}$ with radial distance from the Sun or with solar wind parameters.

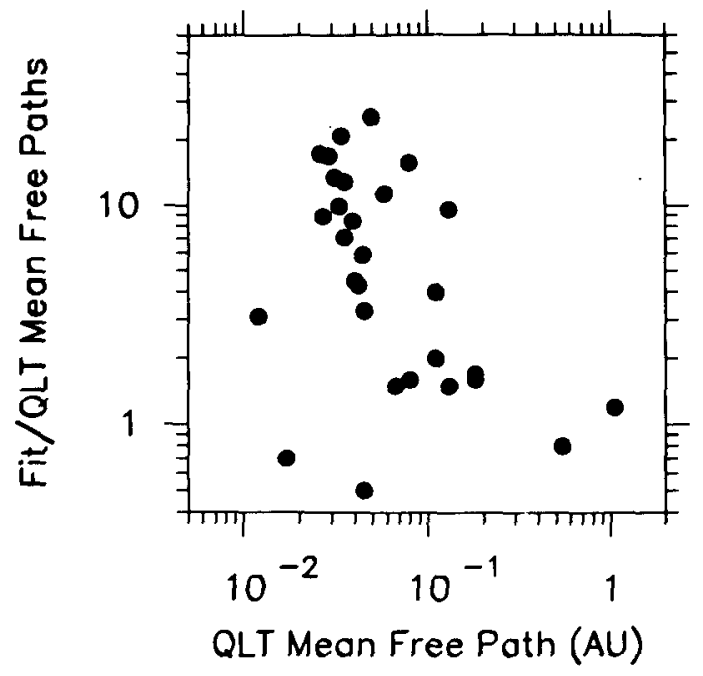

FIG. 3.-Ratio of $\lambda_{\text {fit }}$ for 13-27 MeV protons to $\lambda_{\text {th }}$ derived from magnetic field observation at the same time with standard QLT, as a function of $\lambda_{\text {fit }}$, for 29 selected solar particle events (from Wanner et al. 1993).

The $\lambda_{\mathrm{th}}-\lambda_{\mathrm{fit}}$ discrepancy, together with general doubts in QLT, has led to a large variety of corrections and improvements to the original QLT. Among the proposed mechanisms are mirroring by fluctuations in the magnetic field magnitude (Goldstein, Klimas, \& Sandri 1975), resonance broadening (Völk 1973) and several other nonlinear extensions of the theory (Goldstein 1976; Jones, Birmingham, \& Kaiser 1978), and wave propagation effects (Schlickeiser 1988). The corrections to standard QLT are generally small at large values of $\mu$, but the scattering at values near $\mu=0$ is considerably enhanced. As a consequence, finite mean free paths can be obtained for isotropic turbulence geometries, whereas for slab geometry the discrepancy is further enhanced.

More recent work (Bieber, Smith, \& Matthaeus 1988) has emphasized the presence of dissipation processes in the highwavenumber regime of the fluctuation spectrum which governs the resonant scattering of particles with pitch angles close to $90^{\circ}$. The fact that the spectrum steepens at high wavenumbers had generally been overlooked in earlier attempts to compute $\lambda_{\mathrm{th}}$ which assumed that the inertial range spectrum continues to arbitrary high wavenumbers, and was first considered by Davila \& Scott (1984). Due to the strong suppression of power in the dissipation range, the scattering rate in magnetostatic turbulence vanishes at least as fast as $|\mu|^{2}$ as $\mu \rightarrow 0$. This leads to a nonintegrable singularity at $\mu=0$ in equation (3), regardless of the turbulence geometry. As a result, the predicted mean free path is infinite in resonant magnetostatic QLT for both isotropic and slab fluctuation models (Smith, Bieber, \& Matthaeus 1990). These findings offer new possibilities for reaching a better understanding of particle scattering in the interplanetary medium. Higher order processes, which, as mentioned above, enhance the scattering through the gap at $\mu=0$, are now required to bring back the mean free path from infinity and reconcile theory with observations no matter what the detailed properties of the fluctuations are. In the remainder of this section we will in turn discuss a number of recently 
suggested models which properly take into account the presence of dissipation range spectra and describe particle scattering in either the wave or the turbulence approach.

\subsection{Wave Model: Cold Plasma}

The model proposed by Dröge et al. (1993) considers resonant particle scattering by transversal Alfvén, whistler, and ion and electron cyclotron waves propagating parallel and antiparallel to the large-scale magnetic field $\boldsymbol{B}_{0}$. Because this model was applied to particle observations, and to introduce the basic ideas of wave propagation effects, we will discuss it here in some detail. As was shown by Schlickeiser (1988), wave propagation effects give finite mean free paths for fluctuation spectra steeper than $q=2$ and therefore allow an adequate theoretical treatment of dissipation range spectra. It is assumed that the dispersion relation in a cold plasma holds, and the plasma temperature is only taken into account by assuming a steepening of the high-frequency turbulence spectrum due to damping effects. Scattering by off-axis waves is neglected in this model. The choice of a slab geometry for the magnetic field fluctuations is well supported by the above-mentioned minimumvariance direction parallel to $\boldsymbol{B}_{0}$, and from the fact that oblique propagating MHD waves are quickly damped, depending on the plasma- $\beta$ of the background plasma (Barnes 1979; Achterberg 1981). The pitch-angle diffusion coefficient is then given by

$$
\begin{aligned}
D_{\mu \mu}(\mu)= & \frac{\pi \Omega^{2}}{2 v B_{0}^{2}}\left(1-\mu^{2}\right) \\
& \times\left[\frac{I_{L}^{+}\left(k_{\mathrm{res}}\right)}{|\mu-\epsilon|}+\frac{I_{L}^{-}\left(k_{\mathrm{res}}\right)}{|\mu+\epsilon|}+\frac{I_{R}^{+}\left(k_{\mathrm{res}}\right)}{|\mu-\epsilon|}+\frac{I_{R}^{-}\left(k_{\mathrm{res}}\right)}{|\mu+\epsilon|}\right],
\end{aligned}
$$

where $\epsilon$ is the ratio of the waves' group velocity to the particle velocity, and $I_{R, L}^{+,-}(k)$ are the four (one-sided) power spectra of forward $(+)$ and backward $(-)$ propagating waves with righthand $(R)$ or left-hand $(L)$ polarization. It is convenient to express these four spectra in terms of the cross and magnetic helicity of MHD turbulence theory (cf. Matthaeus \& Goldstein 1982). Introducing the total wave intensity $I_{\text {tot }}(k)$ as the sum of the four spectra, the cross-helicity $h_{c}=\left(I_{R}^{+}+I_{L}^{+}\right) / I_{\text {tot }}$ gives the fractional abundance of forward-propagating waves, whereas the magnetic helicity $\sigma=\left(I_{L}^{+}+I_{L}^{-}-I_{R}^{+}-I_{R}^{-}\right) / I_{\text {tot }}$ describes the net circular polarization of the waves.

The scattering coefficient (eq. [6]) has to be evaluated at the resonant wavenumber $k_{\text {res }}$, which for particles of positive charge to interact with circularly polarized waves is determined by the solution of $(\omega, k \geq 0)$

$$
\omega\left(k_{\mathrm{res}}\right)-s_{1} \mu v k_{\mathrm{res}}=s_{2} \Omega
$$

with $s_{1}=+(-) 1$ for the forward (backward) propagating waves, and $s_{2}=+(-) 1$ for the left-hand (right-hand) waves. The wave frequency is determined by the dispersion relation of the parallel (to $\boldsymbol{B}_{0}$ ) plasma waves (cf. Stix 1962) in the rest frame of the plasma.

As an important special case we notice that at pitch angle $\mu=0$ protons can only interact with left-hand waves, while electrons interact only with right-hand polarized waves. The situation is more complicated at finite $\mu$ (Achatz et al. 1993): most important, if waves of both polarization states propagating in both directions are present, there is no resonance gap at any pitch angle either for electrons or for protons, i.e., these particles find waves to interact with at all pitch angles. While protons can resonantly interact with Alfvén and ion cyclotron waves, electrons can interact with up to three right-hand waves and at least one left-hand wave encompassing Alfvén, whistler, and electron and ion cyclotron waves.

The essential physics of the process of resonant scattering can be illustrated by considering a graphical solution of the resonance condition (for details cf. Davila \& Scott 1984). As an example, we show schematically in Figure 4 the graphical solution for the resonance of protons with forward-propagating left-hand waves. The resonant wavenumber is given by the intersection of the dispersion relation and the resonance condition (eq. [7]), shown for $\mu=0$ and $\mu=-1$. Resonant scattering is possible for pitch angles $-1 \leq \mu \leq \delta$, where $\delta$ is a small (positive) number which goes to zero as the Lorentz factor approaches unity. Protons with $-\delta \leq \mu \leq 1$ can interact with backward-propagating left-hand waves (not shown), so that the full pitch-angle range is covered.

It is obvious from Figure 4 that for nonrelativistic protons ( $\gamma$ close to unity) the value of $k_{\text {res }}$ for scattering through $\mu=0$ which determines $\lambda_{\text {th }}$ depends sensitively on the form of the dispersion relation. Compared with earlier models with propagating waves (Schlickeiser 1988) which assumed a fluctuation spectrum without a dissipation range and a linear dispersion relation (curve $a$ in Fig. 4), the effect of using the exact dispersion relation in a cold plasma and the shape of a dissipation range spectrum in the model of Dröge et al. (1993) is twofold:

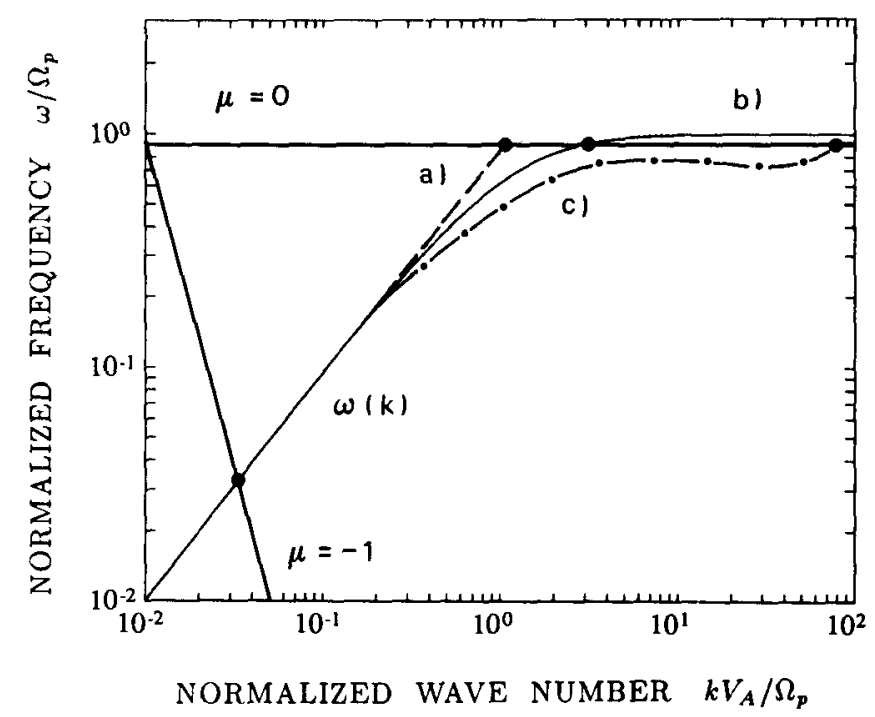

FIG. 4.-Graphical solution of the resonance condition for protons and forward-propagating left-hand polarized waves. Solutions to the dispersion relation $\omega(k)$ are given for $(a)$ linear approximation (dashed line), $(b)$ cold plasma ( solid line), and $(c)$ warm $(\beta=0.3)$ plasma (dash-dot line). The resonance condition (heavy solid line) appears as a straight line with a slope proportional to $\mu$ and intercept $1 / \gamma$. Protons with $-1 \leq \mu \leq 0$ can resonantly interact with these waves. The resonant wavenumber for $\mu=0$ (intersection between the two curves), and thus the mean free path, depend critically on the form of $\omega(k)$ in the dissipation range. 
$k_{\text {res }}(\mu=0)$ is usually in the part of the fluctuation spectrum where the dissipation has already become effective, and applying the correct dispersion relation (curve $b$ in Fig. 4) shifts $k_{\text {res }}$ even more into the dissipation range. As a result, a drastically reduced, while still finite, scattering through $\mu=0$ can be expected, leading to systematically larger values of $\lambda_{\text {th }}$ which are in better agreement with observations.

For three solar particle events observed on Helios where simultaneous particle, magnetic field, and plasma data were available in good quality, values of $\lambda_{\text {fit }}$ were compared with corresponding values of $\lambda_{\text {th }}$ derived from measurements of the Helios fluxgate magnetometer during the time periods of the events. The values of $\lambda_{\mathrm{fit}}$ as a function of rigidity for the events of 1977 November 22 (Helios l, $r=0.63$ AU), 1977 December 27 (Helios 2, $r=0.9 \mathrm{AU}$ ), and 1978 April 11 (Helios $2, r=0.5 \mathrm{AU}$ ) were taken from Beeck et al. (1987) and ValdésGalicia et al. (1988). For the time period spanning 1 day before to 1 day after the onset of the events power spectra $P_{z}\left(f^{\prime}\right)$ of the magnetic field component $B_{z}$ (perpendicular to the ecliptic plane) were constructed using standard methods. Because spectral data were available only in the frequency range from $10^{-4}$ to $6 \times 10^{-2} \mathrm{~Hz}$ for the three events, some assumptions for the continuation of the spectra toward higher wavenumber have to be made. It was assumed that the intensity spectra of the four wave modes are of similar shape in wavenumber space and can be described by double power laws of the form

$$
I_{R, L}^{+,-}(k)=I_{R, L}^{+,-}\left(k_{0}\right) \frac{k^{-q_{1}}}{\left[1+\left(k / k^{*}\right)^{n}\right]^{\left(q_{2}-q_{1}\right) / n}},
$$

where the $I_{R, L}^{+,-}\left(k_{0}\right)$ determine the relative contribution of the respective wave mode, $k^{*}$ is a certain wavenumber where the spectral index $q_{1}$ changes to $q_{2}$, and $n$ models the sharpness of the transition from $q_{1}$ to $q_{2}$. The spectral indices $q_{1}$ at low wavenumbers are then determined by fits to the inertial range spectra, whereas $q_{2}$ and $k^{*}$ are considered as free parameters, although with the restriction that they have the same values for all four spectra. The cross helicity $h_{c}$ is the third free parameter, and the magnetic helicity $\sigma$ is assumed to vanish, as is consistent with observations. The spectra are then transformed individually to frequency space taking into account the Doppler effect. Further conditions are that the spectra are properly normalized, i.e., $\int d k I_{\text {tot }}(k)=2 \int d f^{\prime} P_{z}\left(f^{\prime}\right)=2 \sigma_{z}^{2}$, and that the sum of the spectra is consistent with the observed spectrum at low frequencies and the general spectral form (cf. Fig. 2) at high frequencies.

Mean free paths $\lambda_{\text {th }}$ were calculated varying $h_{c}, q_{2}$, and the cutoff wavenumber, expressed in the dimensionless form $\tilde{k}^{*}=$ $k^{*} V_{\mathrm{A}} / \Omega_{p}$, within the ranges typically observed. It is found that similar results for $\lambda_{\text {th }}$ can be produced by a large variety of different combinations of the three parameters. To apply the theory to particle observations, $\tilde{k}^{*}=2.5$, estimated from the spectra of Denskat et al. ( 1983), was kept constant, and only $q_{2}$ and $h_{c}$ were varied.

In Figure $5 \lambda_{\mathrm{th}}$ is compared with $\lambda_{\mathrm{fit}}$ as a function of rigidity for the three solar events. The parameters assumed were in the range $3 \leq q_{2} \leq 4$ and $0.61 \leq h_{c} \leq 0.84$. Choosing slightly smaller values for $\tilde{k}^{*}$ which were also consistent with typical spectra would require smaller values of $h_{c}$. Values of $\tilde{k}^{*}<2$

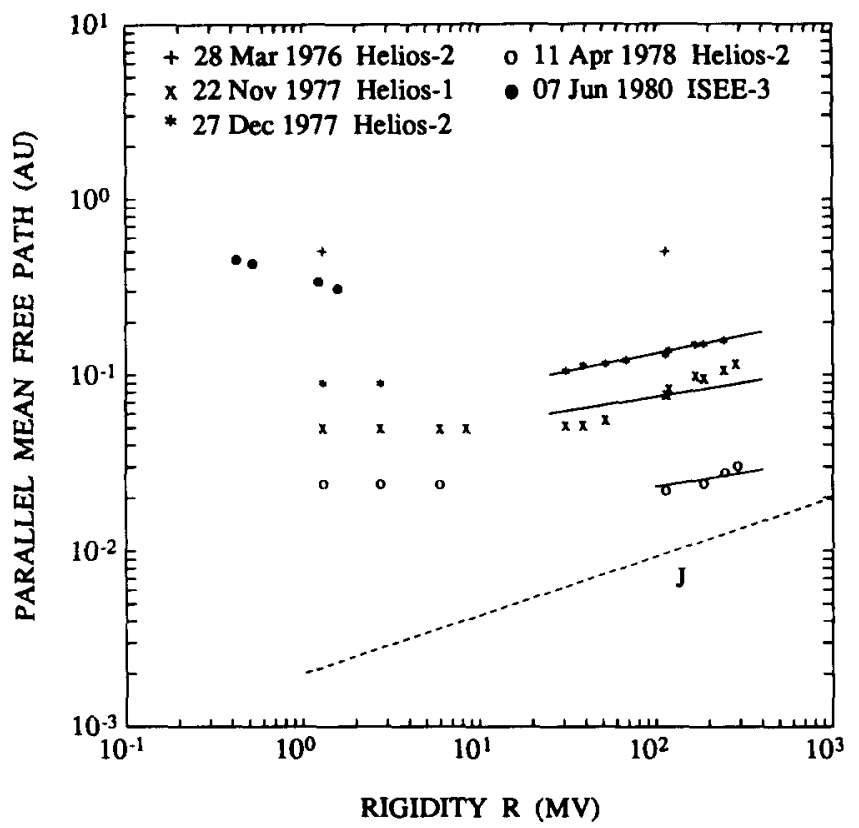

FIG. 5.-Parallel mean free path vs. particle rigidity for selected solar particle events (for source references see text). Dashed line with label "J" represents the prediction of standard QLT based on typical solar wind conditions (Jokipii \& Coleman 1968). Solid lines are predictions of the model of Dröge et al. (1993) derived from magnetic field measurements at the times of the events.

would already make $\lambda_{\text {th }}$ too large for any realistic values of $h_{c}$ and $q_{2}$. Values of $\lambda_{\text {th }}$ which are a factor of 3 larger compared with standard QLT can be achieved well within the observed range of parameters and without any artificial assumptions. The agreement between the two sets of data is remarkably good. For the event of 1977 December 27 the fit is excellent regarding the absolute height as well as the rigidity dependence. For the other two events, the calculated rigidity dependence is somewhat weaker than that obtained from the particle observations.

\subsection{Wave Model: Warm Plasma}

The effects of wave propagation in a warm plasma on the interaction between waves and energetic particles have been studied by Achatz et al. (1993). In their approach they consider solutions of the dispersion relation (e.g., Stix 1962) which can have both real and imaginary parts, $\omega(k)=\omega_{r}(k)+$ $i \Gamma(k)$, where $\Gamma$ is the damping rate. The modifications of finite temperature effects due to a warm Maxwellian background plasma distribution on the cold plasma results can be summarized as follows: oblique-propagating waves are quickly damped, depending on the so-called plasma- $\beta, \beta=8 \pi n_{\kappa} T / B_{0}^{2}$. Parallel-propagating wave modes at low wavenumbers are virtually identical with the ones in a cold plasma $(\beta=0)$. At high wavenumbers left-hand ion cyclotron waves near $\Omega_{p}$ and righthand electron cyclotron waves are strongly cyclotron damped by thermal protons and electrons, respectively. For the ion cyclotron waves Achatz et al. (1993) found that

$$
\Gamma(k) \simeq k v_{\mathrm{th}, p} \exp \left(-\frac{\Omega_{p}}{\beta^{1 / 6} k V_{\mathrm{A}}}\right)
$$


where $v_{\mathrm{th}, p}$ is the thermal background proton velocity. Also, the real part of the frequency of ion cyclotron waves is modified, shown schematically as curve $c$ for the case $\beta=0.3$ in Figure 4. This has important consequences for the resonant calculation of $\lambda_{\mathrm{th}}$. Compared with the cold plasma dispersion relation, the intersection with the resonance condition is shifted toward higher $k$, where there is less power available. As a result, the scattering through $\mu=0$ is drastically reduced for low-rigidity protons, and $\lambda_{\text {th }}$ becomes extremely large below $P \approx 200 \mathrm{MV}$. The fact that this behavior is in contradiction with observations may indicate the existence of processes like nonlinear or thermal resonance broadening or scattering by oblique waves which can enhance the scattering through $\mu=0$. Mean free paths for electrons have also been calculated and compared with data for the 1980 June 7 event observed on ISEE 3 (shown in Fig. 5). Depending on assumptions on the decomposition of the simultaneously observed fluctuation spectra, they are factors of 1.5-10 too large. Again, higher order effects can now help to achieve a better agreement.

\subsection{Turbulence Model plus Mirroring}

Smith (1992) suggests a model which combines resonant scattering theory and mirroring and includes the effect of dissipation range spectra. He assumes that the magnetic fluctuations can be decomposed into two components, one contributing to resonant scattering, while the second is responsible for mirroring. For the mirroring contribution the result of Owens (1974) is used, which leads to resonance line broadening and yields a pitch-angle diffusion coefficient of the form $D_{\mu \mu}^{M}(\mu) \approx$ $C_{1} v\left(1-\mu^{2}\right)$, where $C_{1}$ is related to $\left\langle\delta B^{2}\right\rangle /\left\langle B_{0}^{2}\right\rangle$. The pitchangle scattering coefficient for the combined effects of mirroring and resonant scattering then becomes $D_{\mu \mu}^{\text {tot }}=D_{\mu \mu}^{M}+D_{\mu \mu}^{\text {res }}$. Mean free paths can be determined for both slab and isotropic turbulence, where $D_{\mu \mu}^{\text {res }}$ is calculated assuming typically observed dissipation range spectra with a spectral break around 1 $\mathrm{Hz}$, and the mirroring term acts to yield finite values of $\lambda_{\mathrm{th}}$. The resulting mean free paths show no variation with rigidity below $R \approx 10^{-2}$ and above $10^{5} \mathrm{MV}$, where the contribution of mirroring dominates, and a local minimum of $\lambda_{\mathrm{th}}(R)$ at $\approx 10$ MV. Although no comparisons with particle observations have been made, the general shape of $\lambda_{\mathrm{th}}(R)$ exhibiting a positive slope at $\approx 100 \mathrm{MV}$ rigidities for ions and a zero or negative slope for 0.1-1 MV electrons seems to be in agreement with observations (cf. Fig. 5). Whether this model can predict the correct absolute values of $\lambda_{\mathrm{th}}(R)$ for individual particle events could be tested by measuring $\left\langle\delta B^{2}\right\rangle /\left\langle B_{0}^{2}\right\rangle$ at the times of the events.

\subsection{Dynamical Magnetic Turbulence}

Bieber \& Matthaeus ( 1991 ) introduce the concept of dynamical magnetic turbulence by assuming the magnetic correlation tensor to be of the form $P_{\alpha, \beta}(k, \tau)=P_{\alpha, \beta}^{0}(k, \tau) C(k, \tau)$. Here $P_{\alpha, \beta}^{0}(k, \tau)$ is a valid magnetostatic representation of the turbulence which is considered for both slab and isotropic models with a Kolmogorov $\left(k^{-5 / 3}\right)$ spectrum in the inertial range and a multiplicative factor of $\exp \left(-k / \lambda_{\mathrm{d}}\right)$ representing the dissipation range, with $\lambda_{d}$ the dissipation scale. The dynamical correlation function $C(\boldsymbol{k}, \tau)$ describes a temporal decay process in the two-point correlations and is assumed to be of the form $\exp \left[-t / \tau_{0}(\boldsymbol{k})\right]$, where $\tau_{0}(\boldsymbol{k})$ is the decay time. A probable lower limit for the decay time is estimated to be the Alfven timescale, $\tau_{0}(\boldsymbol{k})=\left(k V_{\mathrm{A}}\right)^{-1}$, so that the dynamical correlation function takes on the form $C(k, \tau)=\exp \left(-\alpha k V_{\mathrm{A}} \tau\right)$, where the parameter $\alpha$ allows us to adjust the strength of the dynamical effects, ranging from $\alpha=0$ (magnetostatic limit) to $\alpha=1$ (strongly dynamical).

Mean free paths were calculated for slab and isotropic geometries using $\alpha=0.1$ and $\alpha=1$. In the slab model with $\alpha=0.1$ the proton mean free path remains almost constant as the rigidity varies from 0.5 to $5000 \mathrm{MV}$, and is a factor of 3 larger compared with standard QLT at $R \approx 100 \mathrm{MV}$. In the isotropic model with the same value of $\alpha$ the difference from the standard model is less pronounced, and even more so for $\alpha=1$ in both geometries. An important feature of the dynamical magnetic turbulence model is that particle scattering depends upon $V_{\mathrm{A}} / v$, leading to different electron and proton mean free paths at the same rigidity. Choosing $\alpha=0.1$, the model predicts a mean free path for $1 \mathrm{MV}$ electrons which is a factor of 100 larger than for $100 \mathrm{MV}$ protons, in obvious contradiction to the fact that observed mean free paths of the two species at these rigidities are approximately similar (Kunow et al. 1991). Recent calculations for $\alpha=1$ (Bieber et al. 1994) and slab geometry give more realistic values for electron mean free paths, although the problem of too small values for $\lambda_{\text {th }}$ remains. To shift $\lambda_{\text {th }}$ closer to the consensus range, the authors suggest that the solar wind fluctuations may be a composite of $20 \%$ slab and $80 \%$ two-dimensional geometries.

\subsection{Wave Model: Resonance Broadening}

Schlickeiser \& Achatz ( 1993 ) investigate the effects of wave damping in a warm plasma on the resonance function in the pitch-angle scattering coefficient. They find similarities between the wave model in $\S 5.2$ and the concept of dynamical magnetic turbulence, in that both approaches lead to pitch-angle scattering coefficients in which the sharp Dirac $\delta$-functions describing the resonant interaction of particles have to be replaced by Breit-Wigner type resonance functions with finite widths into which either the dynamical turbulence decay time or the plasma- $\beta$ enter:

$$
D(k)=\frac{1}{|\mu| v} \frac{\alpha k V_{\mathrm{A}} /(|\mu| v)}{\left[\left(\alpha k V_{\mathrm{A}} /(|\mu| v)\right]^{2}+[k \pm \Omega /(\mu v)]^{2}\right.}
$$

and

$$
\begin{aligned}
D(k) & =\frac{1}{|\mu| v} \\
& \times \frac{\beta^{1 / 2} k V_{\mathrm{A}} /(|\mu| v)}{\left[\beta^{1 / 2} k V_{\mathrm{A}} /(|\mu| v)\right]^{2}+[k \pm \Omega /(\mu v)-\omega /(\mu v)]^{2}} .
\end{aligned}
$$

The widths of the above two resonance functions is determined by the values of $\alpha k V_{\mathrm{A}} /(|\mu| v)$ and $\beta^{1 / 2} k V_{\mathrm{A}} /(|\mu| v)$, respectively. The magnetostatic limit in the dynamical turbulence model is easily recovered by letting $\alpha \rightarrow 0$, in which case the resonance function (eq. [10]) approaches a $\delta$-function with resonance at $k_{\text {res }}=\Omega /(\mu v)$. Likewise, the wave model without thermal wave damping can be reproduced by letting $\beta \rightarrow 0$ in 
equation (11). Including resonance broadening effects in the wave damping model offers the possibility to reduce the mean free paths which are too large due to the modification of only the dispersion relation, and arrive at a consistent explanation of the measurements. The authors claim that the effects of resonance broadening lead to mean free paths which are similar for electrons and protons and approach a constant value at rigidities below $\approx 100 \mathrm{MV}$.

\section{DISCUSSION}

Mean free paths $\lambda_{\text {fit }}$ for a number of selected events where observations over a large range of rigidities are available are shown in Figure 5. Data points below $10 \mathrm{MV}$ are from electrons, and points above $10 \mathrm{MV}$ are from ion measurements. For the three events already discussed in $\$ 5.1$, additional mean free paths have been obtained from four electron channels of the University of Kiel cosmic-ray instruments (Principal Investigator, $\mathrm{H}$. Kunow) on board the Helios spacecraft using a numerical code (Schlüter 1985) for focused particle transport. For the two highest channels only omnidirectional fluxes were available, and anisotropies have been assumed to be similar to the lower energy electrons; in some cases fluxes were not high enough above background to be evaluated. Data for the event of 1980 March 26 were taken from Kunow et al. (1991); electron observations of the 1980 June 7 event are from Achatz et al. (1993).

The key features of the events presented here, which seem to be fairly typical for a larger number of events, are (1) a powerlaw rigidity dependence with slope $\approx 0.3$ for $30-300 \mathrm{MV}$ ions, (2) a constant mean free path for 1-10 MV electrons with approximately the value of $30 \mathrm{MV}$ ions, (3) a decrease of mean free paths with rigidity of $0.4-2 \mathrm{MV}$ electrons possibly marking the transition to the regime of scatter-free transport (Lin 1985 ) for electrons below $\approx 0.1 \mathrm{MV},(4)$ a large variation in the magnitudes of mean free path from one event to another, ranging from 0.02 to $0.5 \mathrm{AU}$ at $100 \mathrm{MV}$, but with little deviation from the general rigidity dependence outlined in items $1-3$, indicating that there are close connections between electron and proton scattering.

The predictions of standard QLT (Jokipii \& Coleman 1968) denoted by curve $J$ in Figure 5 , assuming typical solar wind conditions, are in disagreement with observations in several aspects. The first thing to note is that any comparison between theory and observation should be made on an event-by-event basis; values of $\lambda_{\mathrm{th}}$ derived from fluctuation spectra show as large a variation as values of $\lambda_{\text {fit }}$. Second, observed mean free paths of 30-300 MV ions are on average a factor of 10 larger than standard QLT predictions, whereas the rigidity dependence is qualitatively correct. Electrons exhibit an even larger $\lambda_{\text {fit }}-\lambda_{\text {th }}$ discrepancy and also have a flat rigidity variation compared with the monotonic increase predicted by standard QLT. These two conflicts between theory and observations have been named the "magnitude" and "flatness" problems, respectively, by Bieber et al. (1994). Third, as is evident from the work of Wanner et al. (1993; cf. Fig. 3) and Wanner \& Wibberenz (1993), there also seems to be a "variance" problem: a successful transport theory not only would have to provide systematically larger values of $\lambda_{\mathrm{th}}$ but also must explain the fact that in some events $\lambda_{\text {th }}$ from standard QLT is in rough agreement with $\lambda_{\text {fit }}$, whereas in others it is a factor of $20-30$ too small.

The wave and the dynamical turbulence approaches predict that the mean free path has an explicit velocity dependence which leads to larger mean free paths for electrons than for protons at the same rigidity. Both models therefore offer a possibility of solving the flatness problem. The most promising candidate to solve the magnitude problem, at least if one assumes slab geometry and not that only a fraction of the observed fluctuations contributes to particle scattering, seems to be as of now the wave model in $\S 5.1$, which explains the $\lambda_{\text {fit }}-$ $\lambda_{t h}$ discrepancy in terms of moderate variations of the three parameters characteristic for the fluctuations $h_{c}, \tilde{k}^{*}$, and $q_{2}$. This model can also address the variance problem because minor changes in the three parameters can lead to considerable variations in the resulting mean free paths. It has, however, still to be shown that resonance broadening due to thermal effects can enhance scattering through $\mu=0$ to the right amount, strongly enough to cancel the effects of the modification of the dispersion relation but not strongly enough to remove the effects of the dissipation range spectra totally, which is the case for choosing $\alpha$ or $\beta$ to be of the order of unity.

It seems obvious that mean free paths cannot be predicted from observations of inertial range spectra alone. The mean free path is likely to depend critically on the properties of the fluctuations in the dissipation range: the wavenumber where the spectrum turns over, cross- and magnetic helicities, and the amount of nonresonant scattering at large wavenumbers due to resonance broadening. The latter is controlled by resonance functions whose widths can possibly be estimated from turbulence theory or from measurements of the plasma- $\beta$. Improved observations of magnetic field and plasma parameters in the solar wind with high time resolution would be highly desirable to gain more information about the three-dimensional structure of the magnetic fluctuations. It appears that the theoretical framework is available now to properly take into account higher order corrections to the original QLT and to enable us finally to arrive at a satisfactory description of particle transport and acceleration processes in astrophysical plasmas.

I wish to thank my collaborators U. Achatz, M. Bialk, R. Schlickeiser, W. Wanner, and G. Wibberenz for their cooperation and many stimulating discussions. I am grateful to F. $M$. Neubauer and R. Schwenn for making available Helios magnetic field and plasma data, and to D. Hovestadt, B. Klecker, E. J. Smith, and E. W. Greenstadt for providing ISEE 3 particle, magnetic field, and plasma wave data. 
Bieber, J. W., Matthaeus, W. H., Smith, C. W., Wanner, W., Kallenrode, M.-B., \& Wibberenz, G. 1994, ApJ, in press

Bieber, J. W., Smith, C. W., \& Matthaeus, W. H. 1988, ApJ, 334, 470

Coroniti, F. V., Kennel, C. F., Scarf, F. L., \& Smith, E. J. 1982, J. Geophys. Res., 87,6029

Daily, W. D. 1973, J. Geophys. Res., 78, 2043

Davila, J. M., \& Scott, J. S. 1984, ApJ, 285, 400

Denskat, K. U., Beinroth, H. J., \& Neubauer, F. M. 1983, J. Geophys., 54, 60

Dobrowolny. M., Mangeney, A., \& Veltri, P. 1980, Phys. Rev. Lett., 45, 144

Dröge, W., Achatz, U., Wanner, W., Schlickeiser, R., \& Wibberenz, G. 1993, ApJ, 407, L95

Earl, J. A. 1973. ApJ, 180, 227

Fisk, L. A. 1979, in Solar System Plasma Physics, Vol. 1, ed. E. N. Parker, C. F. Kennel, \& L. J. Lanzerotti (Amsterdam: North-Holland), 177

Fisk, L. A., Goldstein, M. L., Klimas, A. J., \& Sandri, G. 1974, ApJ, 190, 417

Goldstein, M. L. 1976, ApJ, 204, 900

- 1980, J. Geophys. Res., 85, 3033

Goldstein, M. L., Klimas, A. J., \& Sandri, G. 1975, ApJ, 195, 787

Gurnett, D. A. 1991, in Physics of the Inner Heliosphere, Vol. 2, ed. R. Schwenn \& E. Marsch (Heidelberg: Springer), 135

Hasselmann, K., \& Wibberenz, G. 1968, Z. Geophys., 34, 353

Jaekel, U., \& Schlickeiser, R. 1992, J. Phys. G, 18, 1089

Jokipii, J. R. 1966, ApJ, 146, 480

Jokipii, J. R., \& Coleman, P. J. 1968, J. Geophys. Res., 73, 5495

Jones, F. C., Birmingham. T. J., \& Kaiser, T. B. 1978, Phys. Fluids, 21, 347

Krimigis, S. M., \& Roelof, E. C. 1971, J. Geophys. Res., 76, 5921

Kunow, H., Wibberenz, G., Green, G., Müller-Mellin, R., \& Kallenrode, M.-B. 1991, in Physics of the Inner Heliosphere, Vol. 2, ed. R. Schwenn \& E. Marsch (Heidelberg: Springer), 243
Lee, M. A., \& Völk, H.-J. 1975, ApJ, 198, 485

Li, H.-S., \& Zweibel, E. G. 1987, ApJ, 322, 248

Lin, R. P. 1985, Sol. Phys., 100, 537

Marsch, E. 1991, in Physics of the Inner Heliosphere, Vol. 2, ed. R. Schwenn \& E. Marsch (Heidelberg: Springer), 159

Mason, G. M., Gloeckler, G., \& Hovestadt, D. 1984, ApJ, 280, 902

Matthaeus, W. H., \& Goldstein, M. L. 1982, J. Geophys. Res., 87, 6011

Ng, C. K., \& Gleeson, L. J. 1975, Sol. Phys., 43, 475

Owens, A. W. 1974, ApJ, 191, 235

Palmer, I. D. 1982, Rev. Geophys. Space Phys., 20, 335

Parker, E. N. 1965, Planet. Space Sci., 13, 9

Roelof, E. C. 1969, in Lectures in High Energy Astrophysics, ed. H. Ögelmann \& J. R. Wayland (NASA SP-199), 111

Schlickeiser, R. 1988, J. Geophys. Res., 93, 2725

Schlickeiser, R., \& Achatz, U. 1993, J. Plasma. Phys., 49 (Part 1), 63

Schlüter, W. 1985, Ph.D. thesis, Univ. Kiel

Smith, C. W. 1992, in AIP Conf. Proc., Bartol Workshop on Particle Acceleration in Cosmic Plasmas, ed. G. Zank \& T. Gaisser (New York: AIP), 79

Smith, C. W., Bieber, J. W., \& Matthaeus, W. H. 1990, ApJ, 363, 283

Stix, T. H. 1962, The Theory of Plasma Waves (New York: McGraw-Hill) Valdés-Galicia, J. F., Wibberenz, G., Quenby, J. J., Moussas, X., Green, G., \& Neubauer, F. M. 1988, Sol. Phys., 117, 135

Völk, H. J. 1973, Ap\&SS, 25, 47!

Wanner, W., Kallenrode, M.-B., Dröge, W., \& Wibberenz, G. 1993, Adv. Space Res., 13 (No. 9), 359

Wanner, W., \& Wibberenz, G. 1993, J. Geophys. Res., 98, 3513

Zwickl, R. D., \& Webber, W. R. 1977, Sol. Phys., 54, 457 